\title{
Screening of differentially expressed genes in male idiopathic osteoporosis via RNA sequencing
}

\author{
LI FENG $^{1}$, YAN WANG ${ }^{1}$, JING ZHOU $^{2}$, BAOFANG TIAN $^{1}$ and BO XIA ${ }^{1}$ \\ Departments of ${ }^{1}$ Orthopedics and ${ }^{2}$ Gynecology, Jining No. 1 People's Hospital, Jining, Shandong 272011, P.R. China
}

Received October 27, 2017; Accepted March 19, 2018

DOI: $10.3892 / \mathrm{mmr} .2018 .8985$

\begin{abstract}
As a type of osteoporosis (OP), male idiopathic OP (MIO) is a bone disorder that occurs in young males and is a public health problem worldwide. However, the detailed pathogenesis of MIO remains to be elucidated. In the present study, blood samples of patients with MIO, senile OP, postmenopausal OP and normal controls (NCs) were obtained for RNA sequencing. Compared with the NC group, differentially expressed genes (DEGs) in the three types of OP were identified. DEGs that were common among the three types of OP and the DEGs that were unique to patients with $\mathrm{MIO}$ were determined. Gene ontology enrichment analysis and Kyoto Encyclopedia of Genes and Genomes pathway enrichment analyses were conducted. MIO-specific and OP-specific protein-protein interaction (PPI) networks were constructed. Compared with NCs, a total of 519, 368 and 1,472 DEGs were identified in samples from MIO, senile OP and postmenopausal OP, respectively. Tetraspanin 5 (TSPAN5) and $\alpha$-synuclein (SNCA) were unique DEGs in MIO that were not identified in the other two types of OP compared with NCs. Furthermore, the expression of carbonic anhydrase 1 (CA1) and S100 calcium-binding protein P (S100P) in MIO was significantly different compared with senile OP, postmenopausal OP and NC samples. 'MAPK signaling pathway', 'type I diabetes mellitus' and 'hematopoietic cell lineage' were among significantly enriched pathways of DEGs in MIO. SNCA and CDC-like kinase 1 were the hub genes in the
\end{abstract}

Correspondence to: Mr. Bo Xia, Department of Orthopedics, Jining No. 1 People's Hospital, 6 Jiankang Road, Jining, Shandong 272011, P.R. China

E-mail: xiabo1504@163.com

Abbreviations: CA1, carbonic anhydrase 1; CLK1, CDC-like kinase 1; DEGs, differentially expressed genes; GO, gene ontology; HLA, human leukocyte antigen; IGF-I, insulin-like growth factor I; JNK, c-Jun NH2-terminal kinase; KEGG, Kyoto Encyclopedia of Genes and Genomes; MAPK, mitogen-activated protein kinase; OP, osteoporosis; MIO, male idiopathic osteoporosis; NC, normal control; PPI, protein-protein interaction; SNCA, $\alpha$-synuclein; TIDM, type I diabetes mellitus; TSPAN5, tetraspanin 5

Key words: male idiopathic osteoporosis, RNA sequencing, differentially expressed genes, protein-protein interaction network
MIO-specific PPI network. In conclusion, the mitogen-activated protein kinase signaling and type I diabetes mellitus pathways may be involved in bone formation; SNCA and TSPAN5 may be associated with bone resorption. These two pathways and two genes may serve a role in MIO. CA1 and S100P may regulate the process of MIO by modulation of calcification and dysregulation of calcium binding. These findings may have provided an experimental basis for elucidating the underlying mechanisms and developing potential diagnostic biomarkers of $\mathrm{MIO}$.

\section{Introduction}

Osteoporosis (OP) is a common bone disorder that increases the risk of fractures and is characterized by low bone mass $(1,2)$. OP can be classified into primary, secondary and idiopathic types. Primary OP typically affects postmenopausal women and senile men, and is therefore further divided into postmenopausal OP and senile OP (3). OP that occurs in young males with absence of secondary causes of bone loss is termed male idiopathic osteoporosis (MIO) (4). OP results from an imbalance between bone formation by osteoblasts and bone absorption by osteoclasts (5). Decreased recruitment of osteoblasts and failure of vitamin D activation were demonstrated to be associated with senile OP (6). Estrogen deprivation is the primary cause of postmenopausal OP and also serves a role in senile OP (7). Although MIO is an important public health problem worldwide (8), the pathogenesis of MIO differs from the other two types of OP and has not yet been elucidated. However, evidence indicates that osteoblastic defects may lead to MIO (9) and that the insulin-like growth factor I (IGF-I) pathway, and alterations in sex hormones and sclerostin production, also serve roles in the pathogenesis of MIO (9-11). To develop accurate and effective diagnosis and treatment strategies for MIO, the molecular mechanisms underlying the development of this disease requires investigation.

Differentially expressed genes (DEGs) were previously reported to be associated with the pathogenesis of OP, and RNA sequencing is an approach that has been used to identify DEGs in numerous diseases, including OP (12). Using RNA sequencing, the DEGs in patients with $\mathrm{MIO}$, and senile and postmenopausal OP, compared with normal controls (NCs), were identified. DEGs that were common among these three types of OP and unique DEGs only present in patients with MIO were further analyzed. Functional annotation was 
performed and a protein-protein interaction (PPI) network was constructed to further investigate the biological functions of DEGs in OP. To the best of our knowledge, the present study is the first to identify DEGs in patients with MIO by RNA-sequencing. The results of the present study may be used in the future for identification of genes and pathways associated with MIO and aid in elucidating the pathogenesis of this disease.

\section{Materials and methods}

Patients. Patients with OP (2 patients with senile OP, 2 patients with postmenopausal OP and 2 patients with MIO) and two NC patients were recruited from June to August 2016 at Jining No. 1 People's Hospital (Jining, China). Characteristics of patients with MIO and healthy NC patients are presented in Table I. Written informed consent was obtained from all participants and the present study was approved by the Ethics Committee of Jining No. 1 People's Hospital (Jining, China).

RNA isolation and sequencing. Whole blood samples were obtained from all eight participants. Total RNA was isolated from whole blood samples and purified using TRIzol reagent (Invitrogen; Thermo Fisher Scientific, Inc., Waltham, MA, USA) and an RNeasy Mini kit (Qiagen, Inc., Valencia, CA, USA), respectively. The quantity and integrity of purified RNA was verified using an Agilent 2100 Bioanalyzer (Agilent Technologies, Inc., Santa Clara, CA, USA). mRNA that passed quality control (RNA integrity number $>7$ ) was used to construct libraries with a TruSeq RNA Sample Prep kit v2 (Illumina, Inc., San Diego, CA, USA), as described below. Initially, mRNA was fragmented at $95^{\circ} \mathrm{C}$ for 8 min with fragmentation buffer. Subsequently, the fragmented mRNA samples were used as templates for the synthesis of the first cDNA strand using First Strand Master Mix and Super Script II reverse transcriptase (Invitrogen; Thermo Fisher Scientific, Inc.) at $25^{\circ} \mathrm{C}$ for $10 \mathrm{~min}$, $42^{\circ} \mathrm{C}$ for $50 \mathrm{~min}$ and $70^{\circ} \mathrm{C}$ for $15 \mathrm{~min}$. The second cDNA strand was synthesized following the addition of Second Strand Master Mix and incubation at $16^{\circ} \mathrm{C}$ for $1 \mathrm{~h}$. Finally, end repair, adenylate $3^{\prime}$ ends and adapter ligation were performed according to the manufacturer's protocol of the TruSeq RNA Sample Prep kit v2. Polymerase chain reaction (PCR) was performed with PCR Master Mix and RNA PCR primer cocktail in the TruSeq RNA Sample Prep kit v2 (Illumina, Inc., San Diego, CA, USA) to amplify the libraries according to the manufacturer's instructions. The following thermocycling conditions were used for the PCR: Initial denaturation at $98^{\circ} \mathrm{C}$ for $30 \mathrm{sec} ; 11$ cycles of $98^{\circ} \mathrm{C}$ for $10 \mathrm{sec}, 65^{\circ} \mathrm{C}$ for $30 \mathrm{sec}$ and $72^{\circ} \mathrm{C}$ for $15 \mathrm{sec}$, followed by a final extension step of $72^{\circ} \mathrm{C}$ for $10 \mathrm{~min}$. The enriched cDNA libraries were subsequently sequenced using the Illumina HiSeq 2500 (Illumina, Inc.) sequencing platform.

Identification of DEGs. Read QC tool in FastQC version 0.11.4 software (13) was used for the quality control of FASTQ data. Trimming of raw data was performed with cutadapt version 1.9.1 (14). Low quality reads, including adaptor sequences and reads with ratios of $\mathrm{N}$ base $>10 \%$, were removed and clean reads were obtained. TopHat version 2.1.1 (15) was used to align the clean reads to the human genome (GRCh38.p7 assembly, https://www.ncbi.nlm.nih. gov/assembly/GCF_000001405.38) (16). The quantification of mRNA and standardized output was performed using Cuffquant and Cuffdiff in Cufflinks version 2.2.1 (15). Fragments per kilobase of exon per million fragments mapped were used to determine the transcript abundance of each gene. $\mathrm{P}<0.05$ and abs (count_1-count_2) >100 served as the thresholds for the identification of DEGs, where abs indicated the absolute value, and count_1 and count_2 indicated the cases of OP (senile, postmenopausal and $\mathrm{MIO}$ ) and the control group, respectively. The DEGs in MIO, and postmenopausal and senile OP, compared with NCs were identified. DEGs that were common among the three types of OP were identified. A heat map of DEGs in MIO and common DEGs in the three types of OP were generated using the heat map. 2 function in the gplots package in $\mathrm{R}$ version 3.3.3 (17).

Functional annotation. Gene ontology (GO) and Kyoto Encyclopedia of Genes and Genomes (KEGG) pathway enrichment analyses were performed using the online software GeneCodis 3 (http://genecodis.cnb.csic.es/analysis) (18) to interpret the biological function of DEGs in MIO, postmenopausal OP and senile OP. A false discovery rate (FDR) $<0.05$ was considered to indicate a statistically significant difference.

PPI network construction. To determine disease-associated pathways and functions of proteins at the molecular level, the PPI networks of DEGs in MIO, and postmenopausal and senile OP, were constructed using Biological General Repository for Interaction Datasets (http://thebiogrid.org/) and Cytoscape version 3.3.3 (19). Nodes were used to represent proteins and edges to represent interactions between proteins. Based on Cytoscape version 3.3.3, proteins with a degree of $\geq 160$ were defined as hub proteins of the PPI network.

\section{Results}

Identification of DEGs. Compared with NCs, a total of 519 (185 upregulated and 334 downregulated), 368 (272 upregulated and 96 downregulated) and 1,472 (646 upregulated and 826 downregulated) DEGs were identified in MIO, senile OP and postmenopausal OP, respectively. The top 20 upregulated and downregulated DEGs in MIO are presented in Table II.

A total of 138 shared DEGs were identified in the three types of OP compared with NCs. A total of 7 DEGs, including $\alpha$-hemoglobin stabilizing protein (AHSP), carbonic anhydrase 1 (CA1), hemoglobin subunit $\delta$ (HBD), transmembrane and coiled-coil domain family 2 (TMCC2), joining chain of multimeric IgA and IgM (JCHAIN), RP2 ARL3 GTPase-activating protein (RP2) and S100 calcium-binding protein P (S100P), were differentially expressed in MIO compared with both senile and postmenopausal OP (Table III). Certain DEGs, including AHSP, CA1, HBD and TMCC2, were downregulated in MIO but upregulated in senile and postmenopausal OP, compared with NCs. A heat map of the top 100 DEGs in MIO is presented in Fig. 1, while a heat map of the 138 common DEGs among the three types of OP is presented in Fig. 2.

Functional annotation. According to the GO enrichment analysis, 'RNA metabolic process' $\left(\mathrm{FDR}=5.60 \times 10^{-11}\right)$, 


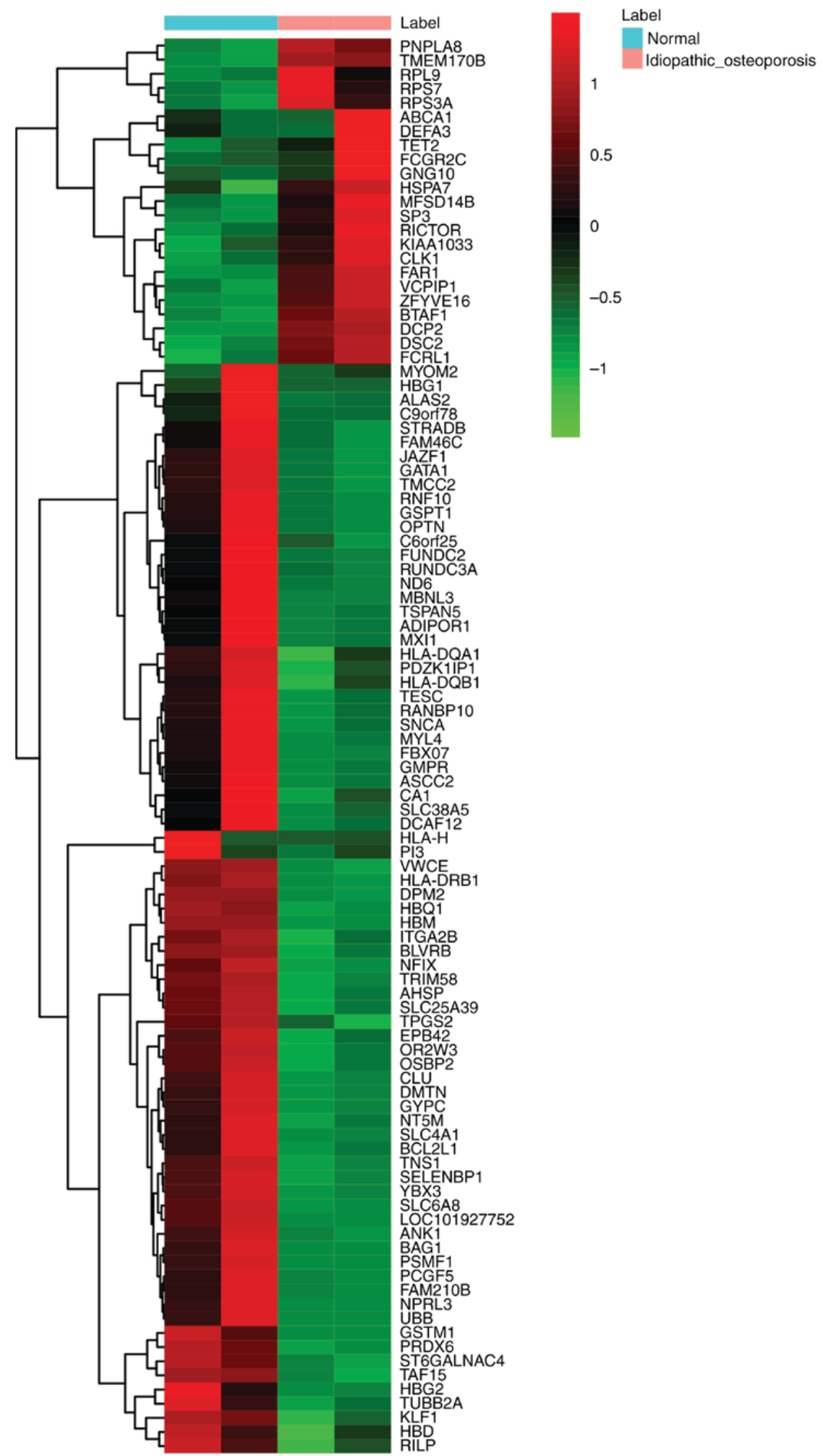

Figure 1. Heat map of top 100 differentially expressed genes in male idiopathic osteoporosis compared with normal controls.

'mRNA metabolic process' (FDR $\left.=8.59 \times 10^{-11}\right)$, 'cytoplasm' $\left(\mathrm{FDR}=9.85 \times 10^{-36}\right)$, 'cytosol' $\left(\mathrm{FDR}=5.98 \times 10^{-34}\right)$, 'protein binding' $\left(\mathrm{FDR}=4.02 \times 10^{-49}\right)$ and 'ATP binding' $\left(\mathrm{FDR}=2.55 \times 10^{-13}\right)$ were the most significantly enriched GO terms of DEGs in MIO (Fig. 3). 'Peptidyl-serine phosphorylation' (FDR $\left.=1.37 \times 10^{-5}\right)$, 'protein phosphorylation' (FDR $\left.=2.43 \times 10^{-5}\right)$, 'cytoplasm' 


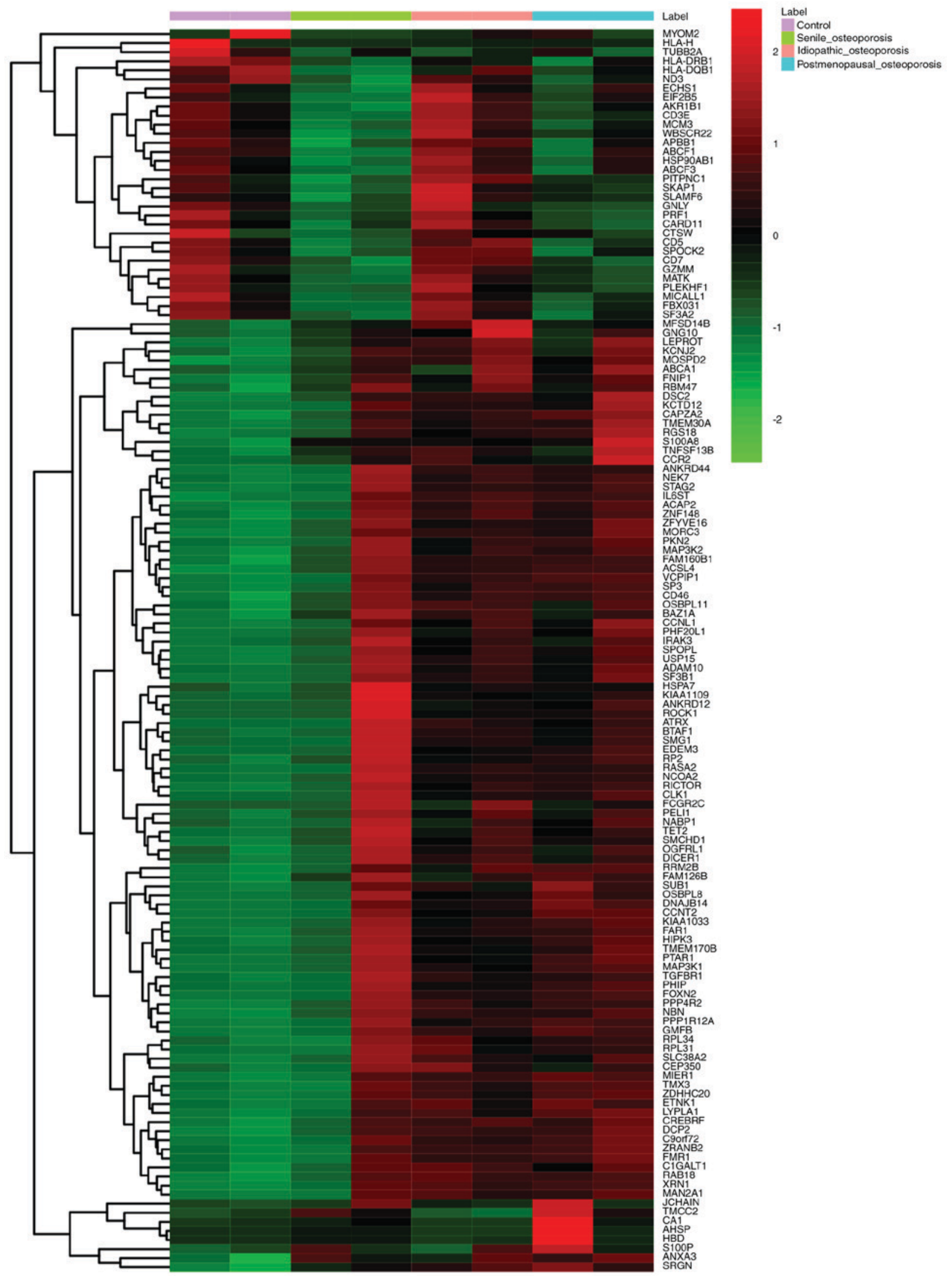

Figure 2. Heat map of 138 common differentially expressed genes among three types of osteoporosis compared with normal controls.

$\left(\mathrm{FDR}=1.82 \times 10^{-8}\right)$, 'nucleus' $\left(\mathrm{FDR}=8.03 \times 10^{-7}\right)$, 'protein binding' $\left(\mathrm{FDR}=4.30 \times 10^{-11}\right)$ and 'ATP binding' $\left(\mathrm{FDR}=3.33 \times 10^{-8}\right)$ were the most significantly enriched GO terms of the 138 common DEGs among the three types of OP (data not shown).
Following KEGG enrichment analysis, 'MAPK signaling pathway' (FDR $\left.=9.25 \times 10^{-4}\right)$, 'type I diabetes mellitus' $\left(\mathrm{FDR}=2.19 \times 10^{-3}\right)$, 'antigen processing and presentation' $\left(\mathrm{FDR}=2.42 \times 10^{-3}\right)$ and 'hematopoietic cell lineage' 

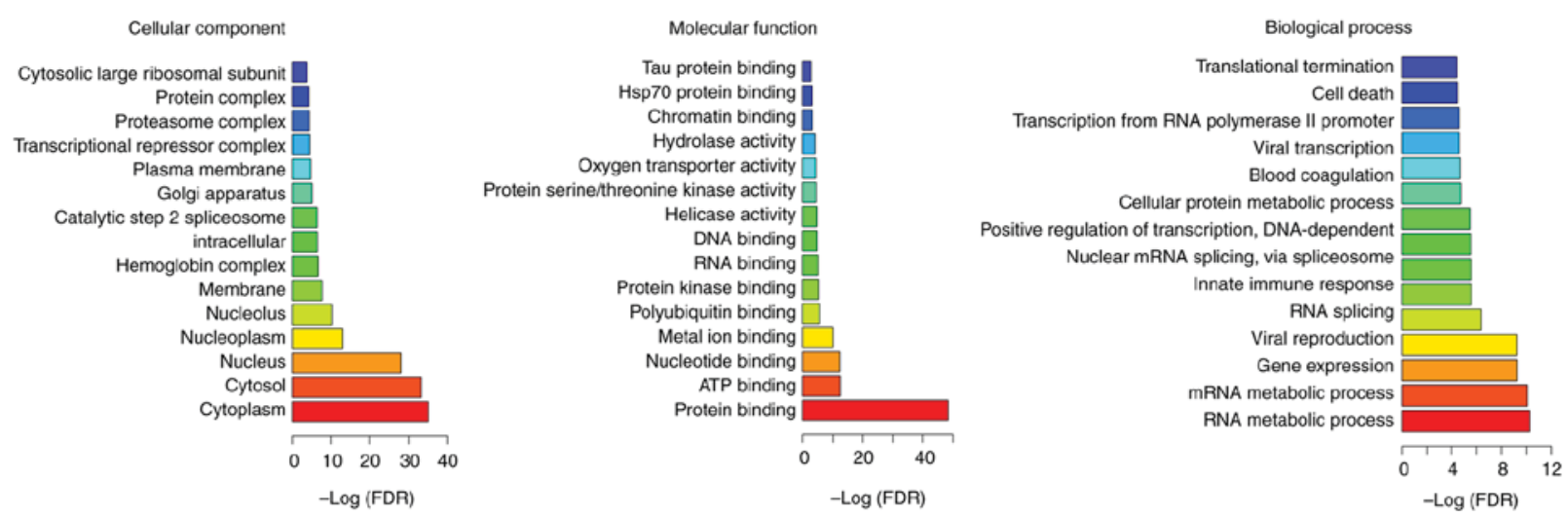

Figure 3. The most significantly enriched gene ontology terms of differentially expressed genes in male idiopathic osteoporosis compared with normal controls. FDR, false discovery rate.

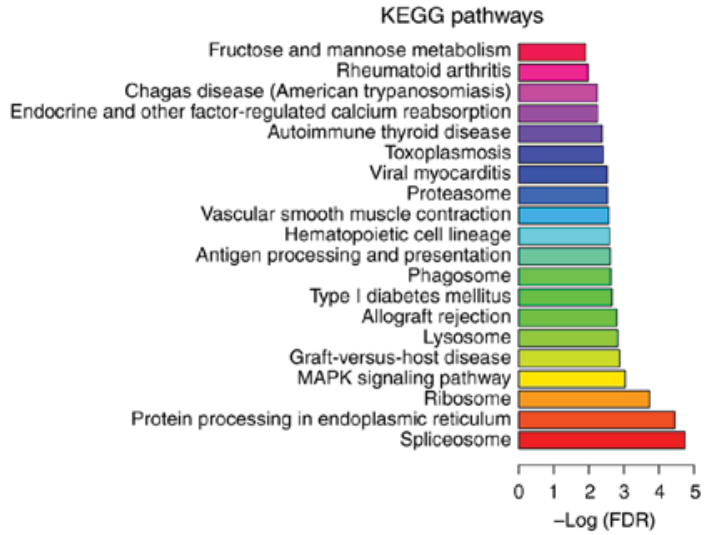

Figure 4. The most significantly enriched KEGG pathways of differentially expressed genes in male idiopathic osteoporosis compared with normal controls. KEGG, Kyoto Encyclopedia of Genes and Genomes; FDR, false discovery rate.

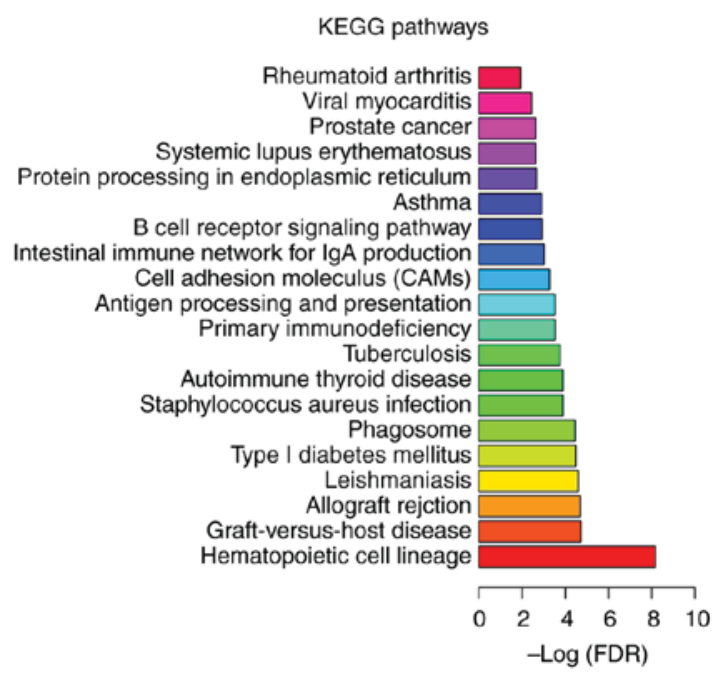

Figure 5. The most significantly enriched KEGG pathways of differentially expressed genes in senile osteoporosis compared with normal controls. KEGG, Kyoto Encyclopedia of Genes and Genomes; FDR, false discovery rate.

$\left(\mathrm{FDR}=2.53 \times 10^{-3}\right)$ were determined to be the most significantly enriched pathways in MIO (Fig. 4). Four DEGs including

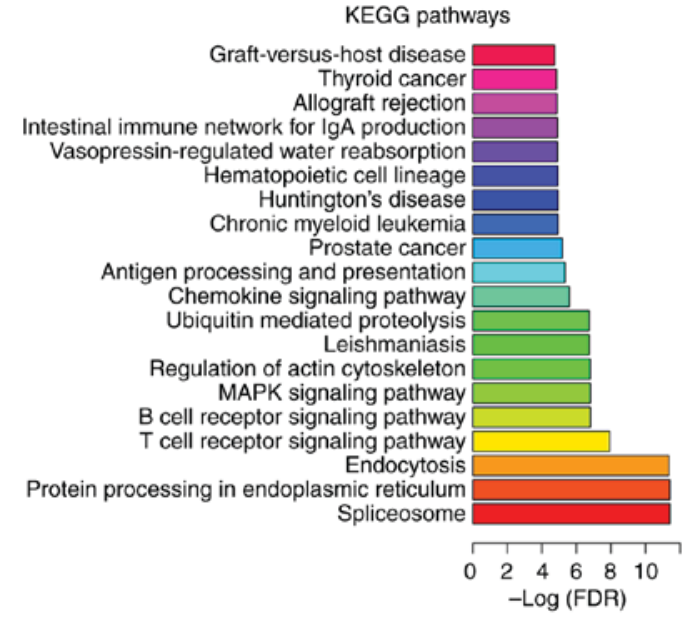

Figure 6. The most significantly enriched KEGG pathways of differentially expressed genes in postmenopausal osteoporosis compared with normal controls. KEGG, Kyoto Encyclopedia of Genes and Genomes; FDR, false discovery rate.

T-cell antigen CD7 (CD7), T-cell surface glycoprotein CD3 $\varepsilon$ chain (CD3E), T-cell surface glycoprotein CD5 (CD5) and major histocompatibility complex class II antigen DR $\beta 1$ (HLA-DRB1) in MIO were significantly enriched in the 'hematopoietic celllineage' pathway.Inaddition, 'hematopoietic cell lineage' (FDR $\left.=6.71 \times 10^{-9}\right)$, 'graft-versus-host disease' $\left(\mathrm{FDR}=1.84 \times 10^{-5}\right)$ and 'allograft rejection' (FDR $\left.=1.98 \times 10^{-5}\right)$ were the most significantly enriched in senile OP (Fig. 5), while 'spliceosome' $\left(\mathrm{FDR}=3.79 \times 10^{-12}\right)$, 'protein processing in endoplasmic reticulum' (FDR=3.83 $\times 10^{-12}$ ) and 'endocytosis' $\left(\mathrm{FDR}=4.41 \times 10^{-12}\right)$ were the most significantly enriched pathways in postmenopausal OP (Fig. 6). 'Hematopoietic cell lineage' was a significantly enriched pathway that was common among all three types of OP (Figs. 4-6).

PPI network construction. The PPI network of the top 20 upregulated and downregulated DEGs in MIO was constructed, which included 1,066 nodes and 1,246 edges (Fig. 7). According to the PPI network, $\alpha$-synuclein (SNCA; degree, 179; clustering coefficient, 0), CDC-like kinase 1 (CLK1; degree, 162; clustering coefficient, 0 ) and receptor tyrosine-protein kinase erbB-2; degree, 160; clustering 
Table I. Patient characteristics.

\begin{tabular}{lcccc}
\hline & MIO & Postmenopausal OP & Senile OP & Normal control \\
\hline No. of patients & 2 & 2 & 2 & 2 \\
Male/female & $2 / 0$ & $0 / 2$ & $2 / 0$ & $2 / 0$ \\
Age (years) & $38.0 \pm 1.0$ & $73 \pm 5.0$ & $89 \pm 0.0$ & $33.5 \pm 1.5$ \\
Smoking history (n) & 2 & 0 & 0 & 0 \\
Alcohol consumption $(\mathrm{n})$ & 2 & 0 & 0 & 2 \\
\hline
\end{tabular}

Data are presented as the mean \pm standard deviation. MIO, male idiopathic osteoporosis.

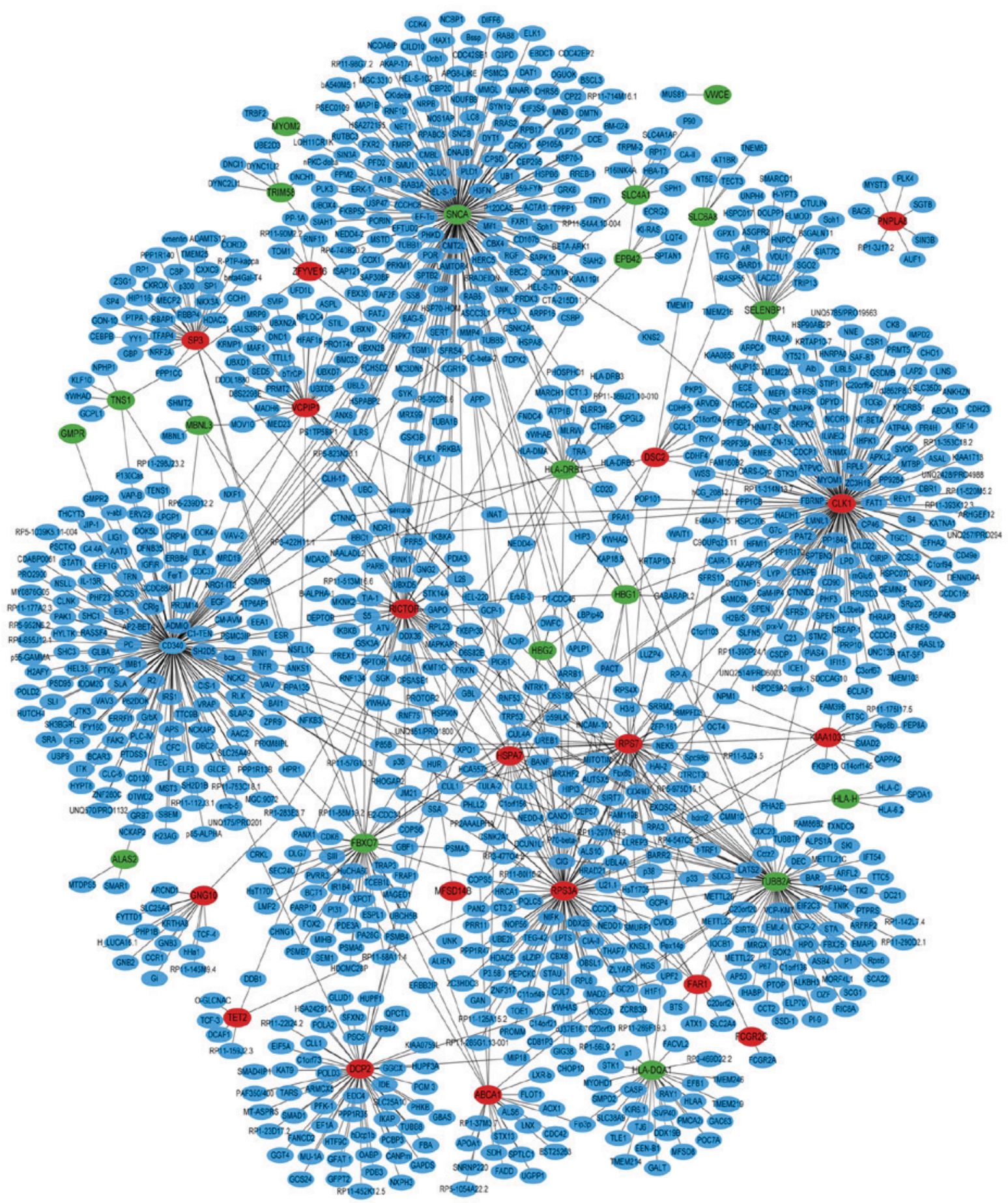

Figure 7. Protein-protein interaction network of top 20 upregulated and downregulated DEGs in male idiopathic osteoporosis compared with normal controls. Red, proteins encoded by upregulated DEGs; green, proteins encoded by downregulated DEGs; blue, other proteins. DEGs, differentially expressed genes. 
Table II. Top 20 downregulated and upregulated differentially expressed genes in patients with male idiopathic osteoporosis compared with normal controls.

A, Downregulated genes

\begin{tabular}{lll}
\hline Gene ID (NCBI) & \multicolumn{1}{c}{ Gene } & P-value \\
\hline 212 & ALAS2 & $5.00 \times 10^{-5}$ \\
2038 & EPB42 & $5.00 \times 10^{-5}$ \\
25793 & FBXO7 & $5.00 \times 10^{-5}$ \\
2766 & GMPR & $5.00 \times 10^{-5}$ \\
3047 & HBG1 & $5.00 \times 10^{-5}$ \\
3048 & HBG2 & $5.00 \times 10^{-5}$ \\
3117 & HLA-DQA1 & $5.00 \times 10^{-5}$ \\
3123 & HLA-DRB1 & $5.00 \times 10^{-5}$ \\
3136 & HLA-H & $5.00 \times 10^{-5}$ \\
55796 & MBNL3 & $5.00 \times 10^{-5}$ \\
9172 & MYOM2 & $5.00 \times 10^{-5}$ \\
8991 & SELENBP1 & $5.00 \times 10^{-5}$ \\
6521 & SLC4A1 & $5.00 \times 10^{-5}$ \\
6535 & SLC6A8 & $5.00 \times 10^{-5}$ \\
6622 & SNCA & $5.00 \times 10^{-5}$ \\
7145 & TNS1 & $5.00 \times 10^{-5}$ \\
25893 & TRIM58 & $5.00 \times 10^{-5}$ \\
10098 & TSPAN5 & $5.00 \times 10^{-5}$ \\
7280 & TUBB2A & $5.00 \times 10^{-5}$ \\
220001 & VWCE & $5.00 \times 10^{-5}$ \\
\hline & & \\
& &
\end{tabular}

$\mathrm{B}$, Upregulated genes

\begin{tabular}{lll}
\hline Gene ID & \multicolumn{1}{c}{ Gene } & P-value \\
\hline 84188 & FAR1 & $5.00 \times 10^{-5}$ \\
9103 & FCGR2C & $5.00 \times 10^{-5}$ \\
84641 & MFSD14B & $5.00 \times 10^{-5}$ \\
253260 & RICTOR & $5.00 \times 10^{-5}$ \\
9765 & ZFYVE16 & $5.00 \times 10^{-5}$ \\
1824 & DSC2 & $1.00 \times 10^{-4}$ \\
3311 & HSPA7 & $1.00 \times 10^{-4}$ \\
2790 & GNG10 & $1.50 \times 10^{-4}$ \\
6670 & SP3 & $2.00 \times 10^{-4}$ \\
23325 & KIAA1033 & $2.50 \times 10^{-4}$ \\
50640 & PNPLA8 & $2.50 \times 10^{-4}$ \\
6201 & RPS7 & $2.50 \times 10^{-4}$ \\
19 & ABCA1 & $3.50 \times 10^{-4}$ \\
167227 & DCP2 & $4.00 \times 10^{-4}$ \\
1195 & CLK1 & $5.00 \times 10^{-4}$ \\
1668 & DEFA3 & $5.00 \times 10^{-4}$ \\
100113407 & TMEM170B & $5.00 \times 10^{-4}$ \\
80124 & VCPIP1 & $5.00 \times 10^{-4}$ \\
6189 & RPS3A & $5.50 \times 10^{-4}$ \\
54790 & TET2 & $5.50 \times 10^{-4}$ \\
\hline & &
\end{tabular}

NCBI, National Center for Biotechnology Information. coefficient, 0) were the 3 hub proteins in MIO. The PPI network of the 138 common DEGs among the three types of OP consisted of 2,680 nodes and 3,957 edges (data not shown). Heat shock protein $90 \alpha$ family class B member 1 (degree, 233; clustering coefficient, $\left.2.22 \times 10^{-4}\right)$, capping actin protein of muscle Z-line $\alpha$ subunit 2 (degree, 229; clustering coefficient, 6.51 $\times 10^{-4}$ ) and CLK1 (degree, 162; clustering coefficient, $1.53 \times 10^{-4}$ ) were the 3 hub proteins. CLK1 was a common hub protein in the MIO-specific PPI network and OP-specific PPI network.

\section{Discussion}

MIO is an important public health problem worldwide (8) and the mechanism underlying this disease remains to be elucidated. The present study identified DEGs in MIO, and senile and postmenopausal OP, compared with NCs. The common DEGs among these three types of OP and unique DEGs only identified in patients with MIO were investigated. The primary symptom of OP is bone loss induced by an imbalance between bone resorption and bone formation. Factors that regulate this balance may be involved in the pathogenesis of OP. Osteoblastic defects have been reported to be associated with the mechanism underlying the development of MIO, and regulators of osteoblasts and bone formation may therefore be associated with MIO.

'MAPK signaling pathway' was a significantly enriched pathway of DEGs in patients with MIO included in the present study. The mitogen-activated protein kinase (MAPK) pathway regulates numerous cellular activities, including cell proliferation, differentiation, survival, death and transformation (20). The MAPK signaling pathway primarily consists of an extracellular signal-regulated kinase, p38 and c-Jun $\mathrm{NH}_{2}$-terminal protein kinase (JNK) (21). Furthermore, the JNK signaling pathway was reported to be associated with the differentiation and proliferation of osteoblasts (22-25). The MAPK signaling pathway and the DEGs enriched in this pathway may serve a role in MIO by regulating bone formation.

Previous studies have demonstrated that IGF-I, which has a similar function and structure to insulin, is crucial for the progression of MIO $(10,26)$. In the present study, DEGs in patients with $\mathrm{MIO}$ were significantly enriched in 'type I diabetes mellitus'. As a deficiency in insulin can impair bone formation induced by osteoblasts, type I diabetes mellitus (TIDM) with low serum IGF-I levels has been reported to be a risk factor for OP (27). Therefore, we hypothesized that the TIDM pathway and its associated DEGs in MIO, including perforin 1, major histocompatibility complex (HLA) class I C, HLA class II DQ $\alpha 1$, HLA class II DR $\beta 1$, HLA class II $\mathrm{DQ} \beta 1$, may serve a role in MIO by regulating bone formation via IGF-I. Furthermore, HLA alleles have been reported to be associated with OP by regulating bone mineral density (28).

In addition to genes involved in the regulation of bone formation by osteoblasts, genes involved in the process of bone resorption by osteoclasts may also be associated with the regulation of bone formation and the mechanism of MIO. Osteoclasts originate from pluripotent hematopoietic cells (5). In the present study, four DEGs (CD7, CD3E, CD5 and HLA-DRB1) in MIO were significantly enriched in the 'hematopoietic cell lineage' pathway, which supported the 
Table III. Differentially expressed genes in MIO compared with NCs and senile and postmenopausal OP.

\begin{tabular}{llllll}
\hline Gene ID (NCBI) & Gene & P-value 1 & P-value 2 & P-value 3 & Differential expression \\
\hline 51327 & AHSP & $5.00 \times 10^{-5}$ & $5.00 \times 10^{-5}$ & $5.00 \times 10^{-5}$ & Downregulated \\
759 & CA1 & $1.00 \times 10^{-4}$ & $5.00 \times 10^{-5}$ & $5.00 \times 10^{-5}$ & Downregulated \\
3045 & HBD & $1.50 \times 10^{-4}$ & $5.00 \times 10^{-5}$ & $5.00 \times 10^{-5}$ & Downregulated \\
3512 & JCHAIN & $1.12 \times 10^{-2}$ & $4.15 \times 10^{-3}$ & $1.50 \times 10^{-4}$ & Upregulated \\
6102 & RP2 & $9.45 \times 10^{-3}$ & $3.48 \times 10^{-2}$ & $2.20 \times 10^{-2}$ & Upregulated \\
6286 & S100P & $2.95 \times 10^{-3}$ & $4.59 \times 10^{-2}$ & $6.00 \times 10^{-4}$ & Upregulated \\
9911 & TMCC2 & $1.00 \times 10^{-4}$ & $2.50 \times 10^{-4}$ & $5.00 \times 10^{-5}$ & Downregulated \\
\hline
\end{tabular}

P-value 1 represents comparisons between the MIO and the NC groups, $\mathrm{P}$-value 2 represents comparisons between the MIO and senile OP groups, and P-value 3 represents comparisons between the MIO and postmenopausal OP groups. The differential expression column represents the upregulation or downregulation of each gene in MIO samples compared with NCs. MIO, male idiopathic osteoporosis; NCs, normal controls; OP, osteoporosis; AHSP, $\alpha$-hemoglobin stabilizing protein; CA1, carbonic anhydrase 1; HBD, hemoglobin subunit $\delta$; JCHAIN, joining chain of multimeric IgA and IgM; RP2, RP2 ARL3 GTPase-activating protein; S100P, S100 calcium-binding protein P; TMCC2, transmembrane and coiled-coil domain family 2; NCBI, National Center for Biotechnology Information.

hypothesis that the number and activity of osteoclasts may be associated with MIO. Furthermore, 'hematopoietic cell lineage' was a common pathway enriched in the other two types of OP, which emphasizes the potential importance of this pathway in OP.

Tetraspanin (TSPAN)5 is a member of the TSPAN family of proteins and was a unique DEG in MIO that was not differentially expressed in the other two types of OP. TSPAN5 was previously reported to be an inhibitor of osteoclastogenesis (24). In the present study, downregulated TSPAN5 was detected in the blood of patients with MIO, which indicated that TSPAN5 may be involved in the process of MIO as reduced TSPAN5 levels leads to the promotion of osteoclastogenesis.

SNCA was another unique DEG in the present study, which was identified only in patients with MIO. SNCA has been demonstrated to be associated with osteoclastogenesis and bone resorption (28) by interacting with the bone morphogenetic protein pathway and modulating bone loss induced by deficiency of estrogen (29-31). In the present study, SNCA was downregulated in patients with $\mathrm{MIO}$ and may serve a role in the development of this disease by regulating bone resorption. Furthermore, SNCA was a hub gene in the MIO-specific PPI network, which emphasizes the potential that this gene may be associated with the pathogenesis of MIO.

In the present study, CLK1 was a hub protein of both the MIO- and OP-specific PPI networks. CLK1 is a dual specificity kinase (31) and was upregulated in all three types of OP, which indicates that CLK1 may regulate the process of OP. However, to the best of our knowledge, an association between CLK1 and OP has not been previously reported. Further studies are required to validate the function of CLK1 in the pathogenesis of OP.

Of the 138 common DEGs in all three types of OP, 7 DEGs (AHSP, CA1, HBD, TMCC2, JCHAIN, RP2 and S100P) were significantly differentially expressed in MIO compared with the other two types of OP. Compared with NCs, CA1 was significantly downregulated in $\mathrm{MIO}$, while it was upregulated in both senile and postmenopausal OP. As a member of the CA family, CA1 has been reported to function in bone formation, bone remodeling and calcification (32). CA1 catalyzes the hydration and dehydration reactions of $\mathrm{CO}_{2} / \mathrm{H}_{2} \mathrm{CO}_{3}$. As $\mathrm{CaCO}_{3}$ is associated with initial bone formation, $\mathrm{CA} 1$ contributes to bone formation and biomineralization by forming a $\mathrm{CaCO}_{3}$ precipitate $(32,33)$. Increased CA1 activity was demonstrated to promote bone resorption (34). Although the mechanism remains to be elucidated, the hydrogen ions produced by CA1 can dissolve the mineral components of bones and may enhance bone resorption (34). By regulating calcification, CA1 serves different roles in bone resorption and formation. As the expression of CA1 in MIO was significantly different compared with the other two types of OP in the present study, it may be hypothesized that CA1 may be involved in the process of MIO by regulating bone mass and that the mechanism may be different compared with that in senile and postmenopausal OP. Further experiments are required to verify this hypothesis.

In the current study, S100P was upregulated in all three types of OP compared with NC. However, the expression of S100P in MIO was markedly decreased compared with senile and postmenopausal OP. S100P is a member of the S100 family of calcium-binding proteins and is a target of bone morphogenetic protein 4 (35). Furthermore, abnormal calcium binding was previously reported to be associated with OP (36), which indicates that S100P may serve a unique role in $\mathrm{MIO}$.

In conclusion, the MAPK signaling pathway and the TIDM pathway may be associated with the process of MIO by regulating bone formation. Furthermore, the hematopoietic cell lineage pathway and two unique DEGs in MIO (SNCA and TSPAN5) may be associated with bone resorption. CA1 and S100P may regulate the process of MIO via functions in calcification and dysregulation of calcium binding. In the present study, CA1 was downregulated in MIO and upregulated in senile and postmenopausal OP, which indicates a unique role for this protein in MIO. However, a lack of biological validation of the results of the current study was a limitation. Further validation with a larger sample sizes, as well as in vitro and in vivo experiments are required to confirm these results. 


\section{Acknowledgements}

Not applicable.

\section{Funding}

No funding was received.

\section{Availability of data and materials}

The datasets used and/or analyzed during the current study are available from the corresponding author on reasonable request.

\section{Authors' contributions}

LF and BX made substantial contributions the conceptualization and design of the study. LF, YW, JZ and BT collected and analyzed the data. LF and YW interpreted the data. All authors were involved in drafting and revising the manuscript and gave final approval of the manuscript.

\section{Ethics approval and consent to participate}

Written informed consent was obtained from all participants and the present study was approved by the Ethics Committee of Jining No.1 People's Hospital (Jining, China).

\section{Consent for publication}

Not applicable.

\section{Competing interests}

The authors declare that they have no competing interests.

\section{References}

1. Karasik D, Rivadeneira F and Johnson ML: The genetics of bone mass and susceptibility to bone diseases. Nat Rev Rheumatol 12 . 496, 2016.

2. Arjmandi BH, Johnson SA, Pourafshar S, Navaei N, George KS, Hooshmand S, Chai SC and Akhavan NS: Bone-protective effects of dried plum in postmenopausal women: Efficacy and possible mechanisms. Nutrients 9: pii: E496, 2017.

3. Usman J and Siddiqui H: Osteoporosis in family practice. J Pak Med Assoc 53: 433-436, 2003.

4. Brumsen C, Papapoulos SE, Lentjes EG, Kluin PM and Hamdy NA: A potential role for the mast cell in the pathogenesis of idiopathic osteoporosis in men. Bone 31: 556-561, 2002.

5. Dudakovic A, Camilleri ET, Riester SM, Paradise CR, Gluscevic M, O'Toole TM, Thaler R, Evans JM, Yan H, Subramaniam M, et al: Enhancer of Zeste homolog 2 inhibition stimulates bone formation and mitigates bone loss caused by ovariectomy in skeletally mature mice. J Biol Chem 291: 24594-24606, 2016.

6. Lee YH, Kim JE, Roh YH, Choi HR, Rhee Y, Kang DR and Lim SK: The combination of vitamin D deficiency and mild to moderate chronic kidney disease is associated with low bone mineral density and deteriorated femoral microarchitecture: Results from the KNHANES 2008-2011. J Clin Endocrinol Metab 99: 3879-3888, 2014.

7. Klein-Nulend J, van Oers RF, Bakker AD and Bacabac RG: Bone cell mechanosensitivity, estrogen deficiency, and osteoporosis. J Biomech 48: 855-865, 2015.

8. Gielen E, Vanderschueren D, Callewaert F and Boonen S: Osteoporosis in men. Best Pract Res Clin Endocrinol Metab 25: 321-335, 2011.
9. Patsch JM, Kohler T, Berzlanovich A, Muschitz C, Bieglmayr C, Roschger P, Resch H and Pietschmann P: Trabecular bone microstructure and local gene expression in iliac crest biopsies of men with idiopathic osteoporosis. J Bone Miner Res 26: 1584-1592, 2011.

10. Paccou J, Dewailly J and Cortet B: Reduced levels of serum IGF-1 is related to the presence of osteoporotic fractures in male idiopathic osteoporosis. Joint Bone Spine 79: 78-82, 2012.

11. Muschitz C, Kocijan R, Pahr D, Patsch JM, Amrein K, Misof BM, Kaider A, Resch H and Pietschmann P: Ibandronate increases sclerostin levels and bone strength in male patients with idiopathic osteoporosis. Calcif Tissue Int 96: 477-489, 2015.

12. Mohammadi Z, Keshtkar A, Fayyazbakhsh F, Ebrahimi M, Amoli MM, Ghorbani M, Khashayar P, Dini M, Ebrahimi-Rad M and Larijani B: Prevalence of osteoporosis and vitamin D receptor gene polymorphisms (FokI) in an Iranian general population based study (Kurdistan) (IMOS). Med J Islam Repub Iran 29: 238, 2016

13. Andrews S: FastQC A Quality Control tool for High Throughput Sequence Data, 2014.

14. Martin M: Cutadapt removes adapter sequences from high-throughput sequencing reads. EMBnet J 17, 2011.

15. Trapnell C, Roberts A, Goff L, Pertea G, Kim D, Kelley DR, Pimentel H, Salzberg SL, Rinn JL and Pachter L: Differential gene and transcript expression analysis of RNA-seq experiments with TopHat and Cufflinks. Nat Protoc 7: 562-578, 2012.

16. Ai H, Ai Y and Meng F: GenomeLandscaper: Landscape analysis of genome-fingerprints maps assessing chromosome architecture. Sci Rep 8: 1026, 2018.

17. Hashemi Gheinani A, Burkhard FC, Rehrauer H, Aquino Fournier C and Monastyrskaya K: MicroRNA MiR-199a-5p regulates smooth muscle cell proliferation and morphology by targeting WNT2 signaling pathway. J Biol Chem 290: 7067-7086, 2015.

18. Tabas-Madrid D, Nogales-Cadenas R and Pascual-Montano A: GeneCodis3: A non-redundant and modular enrichment analysis tool for functional genomics. Nucleic Acids Res 40 (Web Server Issue): W478-W483, 2012

19. Demchak B, Hull T, Reich M, Liefeld T, Smoot M, Ideker T and Mesirov JP: Cytoscape: The network visualization tool for GenomeSpace workflows. F1000Res 3: 151, 2014.

20. Kim EK and Choi EJ: Pathological roles of MAPK signaling pathways in human diseases. Biochim Biophys Acta 1802: 396-405, 2010.

21. Che J P, Li W, Yan Y, Liu M, Wang GC, Li QY, Yang B, Yao XD and Zheng JH: Expression and clinical significance of the nin one binding protein and p38 MAPK in prostate carcinoma. Int J Clin Exp Pathol 6: 2300-2311, 2013.

22. Kim HK, Kim MG and Leem KH: Osteogenic activity of collagen peptide via ERK/MAPK pathway mediated boosting of collagen synthesis and its therapeutic efficacy in osteoporotic bone by back-scattered electron imaging and microarchitecture analysis. Molecules 18: 15474-15489, 2013.

23. Hah YS, Kang HG, Cho HY, Shin SH, Kim UK, Park BW, Lee SI, Rho GJ, Kim JR and Byun JH: JNK signaling plays an important role in the effects of TNF- $\alpha$ and IL-1 $\beta$ on in vitro osteoblastic differentiation of cultured human periosteal-derived cells. Mol Biol Rep 40: 4869-4881, 2013.

24. Zhou J, Fujiwara T, Ye S, Li X and Zhao H: Downregulation of Notch modulators, tetraspanin 5 and 10 , inhibits osteoclastogenesis in vitro. Calcif Tissue Int 95: 209-217, 2014.

25. Kim DY, Kim GW and Chung SH: Nectandrin a enhances the BMP-induced osteoblastic differentiation and mineralization by activation of p38 MAPK-Smad signaling pathway. Korean J Physiol Pharmacol 17: 447-453, 2013.

26. Li J, Zhang L, Zhou L, Yu ZP, Qi F, Liu B, Zi SX, Li L, Li Y, Wang SB, et al: Beneficial effects of non-matched allogeneic cord blood mononuclear cells upon patients with idiopathic osteoporosis. J Transl Med 10: 102, 2012.

27. Yong L, Chengjun H, Jinhua SH and Paul J: Possible linkage between osteoporosis and genes associated with type 1 diabetes mellitus. Med One 1: e170001, 2017

28. Calabrese G, Mesner LD, Foley PL, Rosen CJ and Farber CR: Network analysis implicates alpha-synuclein (Snca) in the regulation of ovariectomy-induced bone loss. Sci Rep 6: 29475, 2016.

29. Bohaty I, Jurásné-Lukovics M, Somogyiné-Vári É, Kozma L, Dankó K and Molnár I: HLA class I, Cw*01 and Cw*15 alleles can play a preventing role in serum IL17 elevation associated with postmenopausal osteoporosis in Hungary. Endocrine, Apr 27, 2013. DOI: 10.1530/endoabs.32.P85. 
30. Alam I, Carr LG, Liang T, Liu Y, Edenberg HJ, Econs MJ and Turner CH: Identification of genes influencing skeletal phenotypes in congenic P/NP rats. J Bone Mineral Res 25: 1314-1325, 2010.

31. Jain P, Karthikeyan C, Moorthy NS, Waiker DK, Jain AK and Trivedi P: Human CDC2-like kinase 1 (CLK1): a novel target for Alzheimer's disease. Curr Drug Targets 15 539-550, 2014.

32. Chang X, Zheng Y, Yang Q, Wang L, Pan J, Xia Y, Yan X and Han J: Carbonic anhydrase I (CA1) is involved in the process of bone formation and is susceptible to ankylosing spondylitis. Arthrit Res Ther 14: R176, 2012.

33. Li W, Chen WS, Zhou PP, Cao L and Yu LJ: Influence of initial $\mathrm{pH}$ on the precipitation and crystal morphology of calcium carbonate induced by microbial carbonic anhydrase. Colloids Surf B Biointerfaces 102: 281-287, 2013.
34. Chang X, Han J, Zhao Y, Yan X, Sun S and Cui Y: Increased expression of carbonic anhydrase I in the synovium of patients with ankylosing spondylitis. BMC Musculoskelet Disord 11: 279, 2010.

35. Tóthová V and Gibadulinová A: S100P, a peculiar member of S100 family of calcium-binding proteins implicated in cancer. Acta Virol 57: 238-246, 2013

36. Li XL, Wu MS, Zhu XW, Deng YC, Ye YY, Zhao SZ, Ren LZ and Li B: Effects of flavonoids from Cuscuta chinensis on intestinal calcium-binding protein mRNA expression in ovariectomized osteoporosis model rats. Chin J Tissue Eng Res: 4271-4276, 2014.

cc)(ㅇ) $\ominus$ This work is licensed under a Creative Commons Attribution-NonCommercial-NoDerivatives 4.0 International (CC BY-NC-ND 4.0) License. 